\title{
NANOPARTÍCULAS DE ÓXIDOS DE MANGANÊS, FERRO E CÉRIO COMO CATALISADORES DA OZONIZAÇÃO DE EFLUENTES DE REFINARIA DE PETRÓLEO
}

\author{
A. P. S. LIMA, G. SCARATTI, J. R. BAKKAR, H. J. JOSÉ e R. F. P. MOREIRA \\ Universidade Federal de Santa Catarina, Departamento de Engenharia Química e Engenharia de \\ Alimentos \\ E-mail para contato: apaulasl.ufsc@gmail.com
}

\begin{abstract}
RESUMO - Efluentes gerados pela indústria petroquímica são de composição química heterogênea e contém substâncias tóxicas e recalcitrantes. $\mathrm{O}$ desenvolvimento de tecnologias para o tratamento desses efluentes é de extrema importância, pois as técnicas convencionais não se mostram adequadas no tratamento efluentes com Demanda Química de Oxigênio (DQO) acima de $1000 \mathrm{mg} \mathrm{L}^{-1}$. As reações de oxidação avançada, com ozônio associado a catalisadores de metais não nobres, mostram-se como uma alternativa viável e são consideradas tecnologias limpas devido a sua alta capacidade de degradar substâncias recalcitrantes. Sendo assim, este trabalho objetivou investigar a cinética de mineralização em um reator descontínuo com nanopartículas de óxidos de ferro, manganês e cério em suspensão aquosa de composição semelhante a um efluente de refinaria de petróleo, assim como caracterizar as nanopartículas quanto a área BET, espectroscopia fotoeletrônica de raio-X e análises termogravimétricas. Os resultados mostraram que os catalisadores utilizados apresentaram boa atividade catalítica, aumentando na ordem $\mathrm{FeOOH}>$ $\mathrm{Mn}_{2} \mathrm{O}_{3} \sim \mathrm{CeO}_{2}$, alcançando remoção de DQO de até $90 \%$ e COT de até $80 \%$ em 5 horas de reação.
\end{abstract}

\section{INTRODUÇÃO}

As principais técnicas convencionais de tratamento de efluentes da indústria petroquímica necessitam de alta demanda de área física, são de alto custo e baixa eficiência quando aplicadas como sistema único de tratamento e, muitas vezes não se enquadram nos padrões de lançamento exigidos pelos órgãos ambientais (Barbosa, 2007; Mariano, 2001).

As reações de oxidação avançada são alternativas viáveis (Diya'uddeen et al., 2011) e são consideradas tecnologias limpas que minimizam a geração de poluição secundária. Nesse sentido, uma das tecnologias mais promissoras é o processo de ozonização associado a catalisadores de metais não nobres. As reações de ozonização podem promover redução na DQO e do teor de Carbono Orgânico Total (COT), porém, geralmente, os níveis de redução de matéria orgânica ainda são menores que os obtidos com outros processos oxidativos. Devido a estas limitações, estudos buscam aumentar a eficiência dos processos de ozonização, principalmente com relação 


\section{9 a 22 de outubro de 2014 \\ Florianópolis/SC}

às taxas de mineralização dos compostos orgânicos poluentes e também tornar o processo mais economicamente viável (Diya'uddeen et al., 2011; Coelho et al.,2006; Mustafa, 1998). Assim, a ozonização catalítica, torna-se uma tecnologia de oxidação promissora quando utilizada na remoção de contaminantes específicos, bem como ao tratamento de efluentes (Park et. al, 2004).

Estudos recentes mostram que a presença de catalisadores durante o processo de ozonização, aumenta a formação de radical hidroxila, o que possibilita um aumento na decomposição ou mineralização de substâncias orgânicas presentes (Almeida et. al, 2004), aumentando assim, o desempenho do processo e reduzindo o consumo de ozônio quando comparado a ozonização convencional (Britto \& Rangel, 2008). Na ozonização catalítica, o catalisador, como uma superfície heterogênea, aumenta a dissolução do ozônio e age como iniciador da reação de decomposição. Portanto, a eficiência da ozonização catalítica depende, principalmente, da quantidade de catalisador utilizado e de suas propriedades de superfície (Kasprzyk-Hordern et al., 2003). O catalisador deve ser ativo, não-seletivo, que degrade qualquer tipo de contaminante presente no efluente, estável em relação às condições do processo e à natureza do substrato, possuir atividade longa e, caso perdê-la, ser possível restaurá-la ao nível inicial (Nogueira, 2009).

Apesar da crescente aplicação da ozonização catalítica, ainda se faz necessário um maior conhecimento em relação aos mecanismos de reação do ozônio com os metais, assim como estudos sobre os catalisadores utilizados.

\subsection{Objetivos}

O objetivo deste trabalho foi avaliar o processo de oxidação catalítica de compostos orgânicos presentes em um efluente sintético de refinaria de petróleo, utilizando ozônio em um reator descontínuo com nanopartículas de catalisadores heterogêneos de óxidos de ferro, manganês e cério, em suspensão aquosa.

\section{MATERIAIS E MÉTODOS}

\subsection{Efluente de refinaria sintético}

Foi preparada solução aquosa com características semelhantes ao efluente líquido de uma refinaria de petróleo, como proposto por Lin e colaboradores (2001). A composição principal de carbono orgânico é ácido benzóico $\left(500 \mathrm{mg} \mathrm{L}^{-1}\right)$ e ácido aminobenzóico $\left(100 \mathrm{mg} \mathrm{L}^{-1}\right)$, juntamente com uma solução de tampão inorgânico, composta de $\left(\mathrm{NH}_{4}\right)_{2} \mathrm{SO}_{4}\left(250 \mathrm{mg} \mathrm{L}^{-1}\right), \mathrm{K}_{2} \mathrm{HPO}_{4}(222 \mathrm{mg}$ $\left.\mathrm{L}^{-1}\right), \mathrm{KH}_{2} \mathrm{PO}_{4}\left(8,5 \mathrm{mg} \mathrm{L}^{-1}\right), \mathrm{NaHPO}_{4} \cdot \mathrm{H}_{2} \mathrm{O}\left(44,6 \mathrm{mg} \mathrm{L}^{-1}\right), \mathrm{NH}_{4} \mathrm{Cl}\left(1,7 \mathrm{mg} \mathrm{L}^{-1}\right), \mathrm{MgSO}_{4} .7 \mathrm{H}_{2} \mathrm{O}(2,25$ $\left.\mathrm{mg} \mathrm{L}^{-1}\right), \mathrm{NaHCO}_{3}\left(800 \mathrm{mg} \mathrm{L}^{-1}\right)$ e $\mathrm{FeCl}_{3}, 6 \mathrm{H}_{2} \mathrm{O}\left(0,03 \mathrm{mg} \mathrm{L}^{-1}\right)$. A DQO dessa solução aquosa é $1146,0 \mathrm{mg} \mathrm{L}^{-1}$ e COT de $410 \mathrm{mg} \mathrm{L}^{-1}$. Esta solução aquosa é designada neste trabalho como ERS.

\subsection{Caracterização dos catalisadores}

Foram utilizadas nanopartículas de goetita, fornecidas pela empresa Carbonífera Criciúma 
S.A. e nanopartículas de $\mathrm{Mn}_{2} \mathrm{O}_{3}$ e de cério, obtidas da empresa SSNANO (Houston, TX, EUA).

2.2.1 Área BET: a área superficial especifica (BET) e distribuição de diâmetro de poros (BJH) foram obtidas por meio das isotermas de adsorção e dessorção de $\mathrm{N}_{2}$ a $77 \mathrm{~K}$, em um equipamento Autosorb-3b BET, Quantachrome (Rice University, Houston,TX, EUA).

2.2.2 Espectroscopia de fotoelétrons de raio-X (XPS): as análises foram realizadas em um equipamento PHI Quantera XPS (Rice University, Houston, TX, EUA), com radiação monocromática de $\mathrm{Al} \mathrm{K} \alpha$ de 1486,6 eV como fonte de raios-X.

2.2.3 Análises Termogravimétricas (ATG): foram realizadas em um analisador termomecânico Shimadzu DSC/DTG 60 (LEMA/EQA/UFSC), em condições de atmosfera de gás inerte $\left(\mathrm{N}_{2}\right)$, fluxo de $100 \mathrm{~mL} \mathrm{~min}^{-1} \mathrm{e}$ taxa de aquecimento de $10^{\circ} \mathrm{C} \mathrm{min}^{-1}$.

\subsection{Tratamento de efluente sintético de refinaria por ozonização catalítica e não catalítica}

Para os experimentos de ozonização não catalítica foi adicionado ao reator 1,5 L de ERS em pH 7,0 e, para os experimentos de ozonização catalítica, foram utilizados os óxidos metálicos em suspensão aquosa com dosagem de catalisador de $0,5 \mathrm{~g} \mathrm{~L}^{-1}$. O reator é operado de forma semi-contínua, pela alimentação contínua de ozônio (gerador de ozônio Philozon $\mathrm{O}_{3} \mathrm{R}$ modelo ID-10, localizado no LEMA/EQA/UFSC), conforme Figura 1. Em intervalos determinados de tempo, uma alíquota do líquido foi retirada e filtrada em membrana de PVDF (Milipore) $(0,22$ $\mu \mathrm{m})$ para a determinação analítica de COT e DQO.

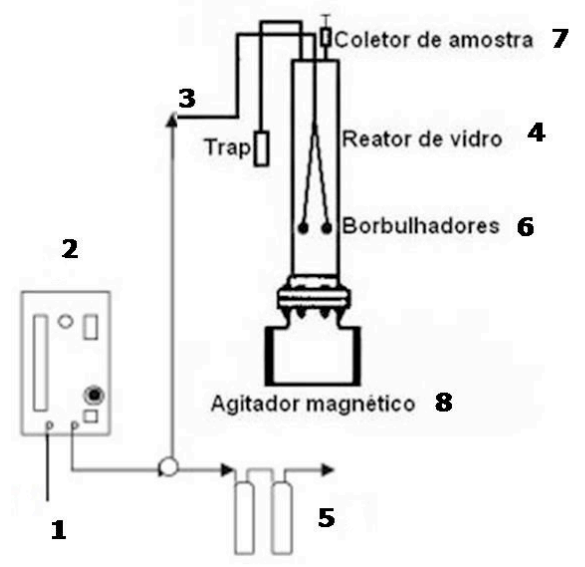

Figura 1 - Esquema do sistema experimental a ser utilizado neste trabalho. 1. Entrada de ar atmosférico; 2: gerador de ozônio; 3. Entrada de ozônio no reator; 4: Reator de 2,0 L; 5: traps com iodeto de potássio; 6. Borbulhadores de ozônio; 7. Ponto de amostragem; 8. Agitador. 


\section{RESULTADOS E DISCUSSÕES}

\subsection{Caracterização dos catalisadores}

A Tabela 1 mostra os resultados obtidos de área superficial BET, o volume total de poros e o diâmetro médio de poros dos catalisadores. As isotermas de adsorção/dessorção de nitrogênio obtidas para todas as amostras foram praticamente idênticas, apresentando histerese pouco significativa. Segundo a IUPAC (1994), as isotermas podem ser classificadas como do tipo III, típica de adsorventes em multicamadas não porosos, macroporosos ou materiais com mesoporos. A distribuição de tamanho médio de poros pelo método $\mathrm{BJH}$, mostrou que o diâmetro de poros das amostras se concentra entre 2 e $10 \mathrm{~nm}$, confirmando as características de materiais mesoporosos.

Tabela 1 - Caracterização textural dos catalisadores

\begin{tabular}{|l|c|c|c|c|}
\hline \multirow{2}{*}{ Catalisador } & \multirow{2}{*}{$\mathrm{S}_{\text {BET }}\left(\mathrm{m}^{2} \cdot \mathrm{g}^{-1}\right)$} & \multirow{2}{*}{$\mathrm{V}_{\mathrm{p}}\left(\mathrm{cm}^{3} \cdot \mathrm{g}^{-1}\right)$} & \multicolumn{2}{|c|}{$\mathrm{D}_{\mathrm{p}}(\mathrm{nm})$} \\
\cline { 4 - 5 } & 55,70 & $2,80 \times 10^{-2}$ & $\mathrm{BET}$ & $\mathrm{BJH}$ \\
\hline Goetita & 15,60 & $7,89 \times 10^{-3}$ & 2,64 & 2,92 \\
\hline $\mathrm{Mn}_{2} \mathrm{O}_{3}$ & 51,40 & $2,60 \times 10^{-2}$ & 2,63 & 3,78 \\
\hline $\mathrm{CeO}_{2}$ & & & \\
\hline
\end{tabular}

$\mathrm{S}_{\mathrm{BET}}=$ área superficial específica, $\mathrm{V}_{\mathrm{p}}=$ volume total de poros, $\mathrm{D}_{\mathrm{p}}=$ diâmetro médio de poros.

As medidas de XPS forneceram informações sobre o estado de oxidação das nanopartículas. A Figura 2a, 2b e 2c apresenta o espectro O (1s) dos catalisadores. Foi detectada uma alta percentagem de oxigênio em todas as amostras, como esperado. Parte desse oxigênio é proveniente do $\mathrm{O}_{2}$ adsorvido na amostra, por isso são obtidos dois picos de oxigênio. A goetita (Figura 2a) apresentou concentração atômica de $\mathrm{O}(1 \mathrm{~s})$ de $82,41 \%$ e Fe $\left(2 \mathrm{p}_{3 / 2}\right)$ de $17,59 \%$, o $\mathrm{CeO}_{2}$ (Figura 2b) $80,99 \%$ para $\mathrm{O}(1 \mathrm{~s})$ e $19,01 \%$ para $\mathrm{Ce}\left(3 \mathrm{~d}_{5 / 2}\right)$ e o $\mathrm{Mn}_{2} \mathrm{O}_{3}$ (Figura 2c) apresentou $65,96 \%$ para O (1s) e $34,04 \%$ para $\mathrm{Mn}\left(2 \mathrm{p}_{3 / 2}\right)$.

Para a goetita, Figura $2 \mathrm{~d}$, a presença do pico de $\mathrm{Fe}\left(2 \mathrm{p}_{3 / 2}\right)$ com energia de ligação em 711 $\mathrm{eV}$, indica que o ferro está no estado de oxidação Fe (III) (Biesinger et. al., 2011). O cério, Figura 2e, apresenta dupletos em aproximadamente, 898 e $917 \mathrm{eV}$, identificados como $v^{\prime \prime \prime}$ e u'", respectivamente, indicando a presença de Ce (IV) (Mullins et. al., 1998). O manganês, Figura 2f, apresenta picos em aproximadamente 641 e $653 \mathrm{eV}$, que são referentes a $\mathrm{Mn} 2 \mathrm{p}_{3 / 2}$ e $\mathrm{Mn} 2 \mathrm{p}_{1 / 2}$, respectivamente e indicam a existência de Mn (III) (Biesinger et. al., 2011). 
(a)

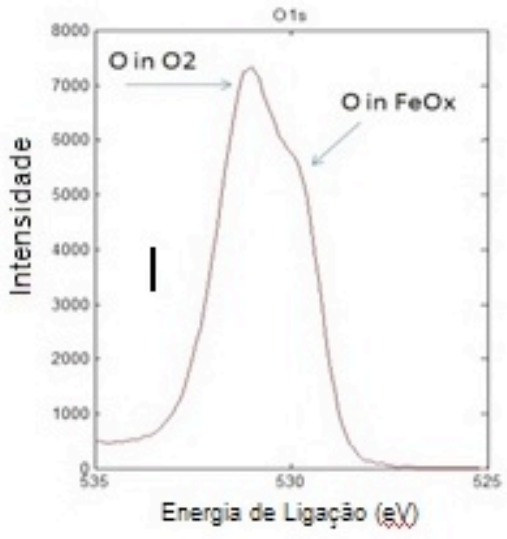

(d)

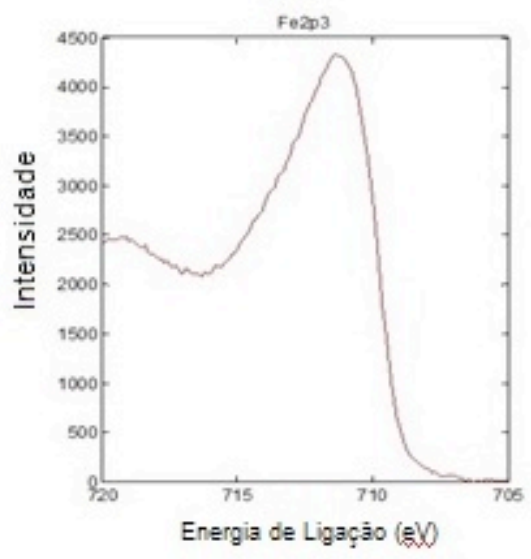

(b)

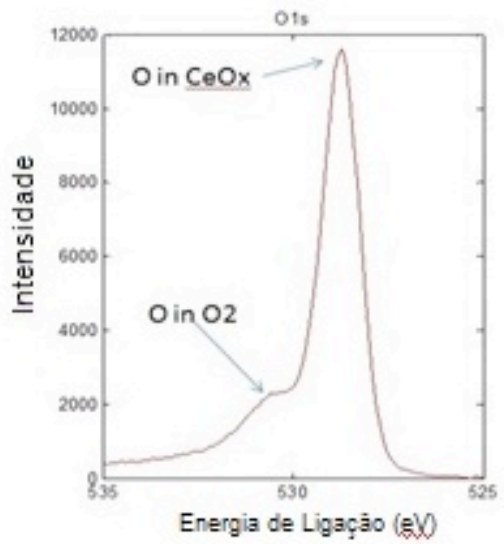

(e)

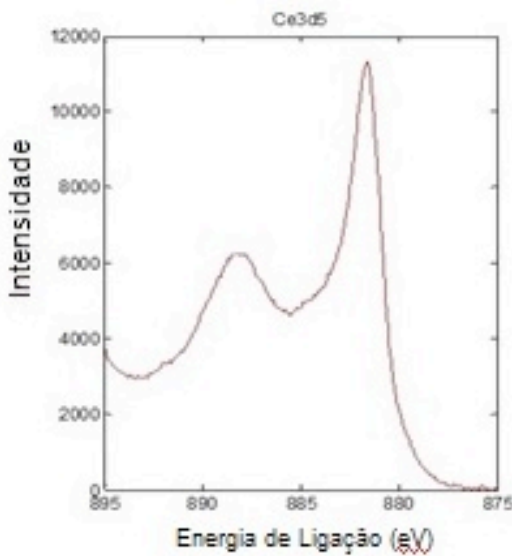

(c)

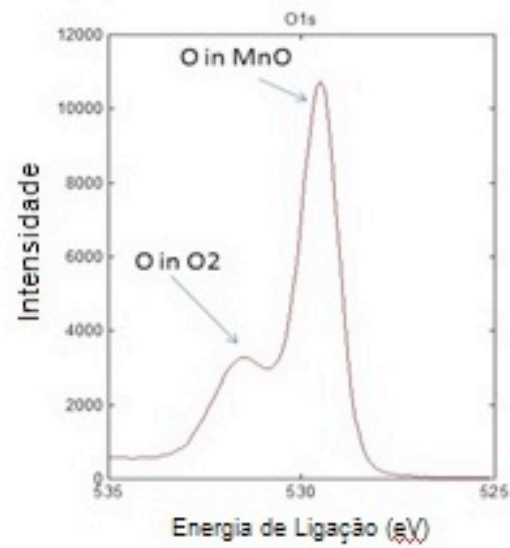

(f)

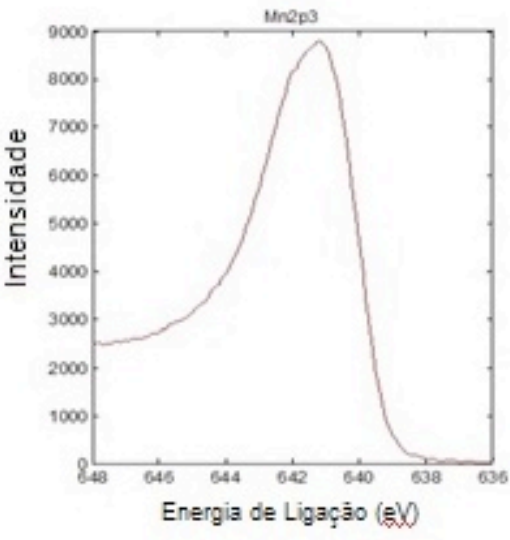

Figura 2 - Espectro XPS das nanopartículas: (a) e (d) Goetita, (b) e (e) $\mathrm{CeO}_{2}$ e (c) e (f) $\mathrm{Mn}_{2} \mathrm{O}_{3}$.

A análise termogravimétrica da goetita (Figura 3a) indicou dois picos endotérmicos relacionados à perda de massa. $\mathrm{O}$ primeiro na temperatura de $102{ }^{\circ} \mathrm{C}$, associado à perda de água da superfície e o segundo, na temperatura de $260^{\circ} \mathrm{C}$, associado a presença de grupos hidroxila e transformação da goetita em hematita, que ocorre entre 260 a $320^{\circ} \mathrm{C}$ (Cornell e Schwertmann, 2003). Acima de $300^{\circ} \mathrm{C}$ ocorre a liberação de moléculas de água devido à formação da hematita e, em temperaturas maiores que $600{ }^{\circ} \mathrm{C}$, a perda de massa é atribuída a eliminação de sulfatos, que está presente nesta amostra (Cornell e Schwertmann, 2003). A Figura 3b mostra a análise ATG de $\mathrm{CeO}_{2}$, indicando que o tratamento térmico aplicado na produção deste catalisador eliminou quase todo o teor de grupos hidroxila e água da superfície dos sólidos. A Figura 3c mostra a análise de ATG para o $\mathrm{Mn}_{2} \mathrm{O}_{3}$, indicando que a partir de $675^{\circ} \mathrm{C}$ ocorre a transformação de $\mathrm{Mn}_{2} \mathrm{O}_{3}$ em $\mathrm{Mn}_{3} \mathrm{O}_{4}$ (Ding et. al., 2005). 

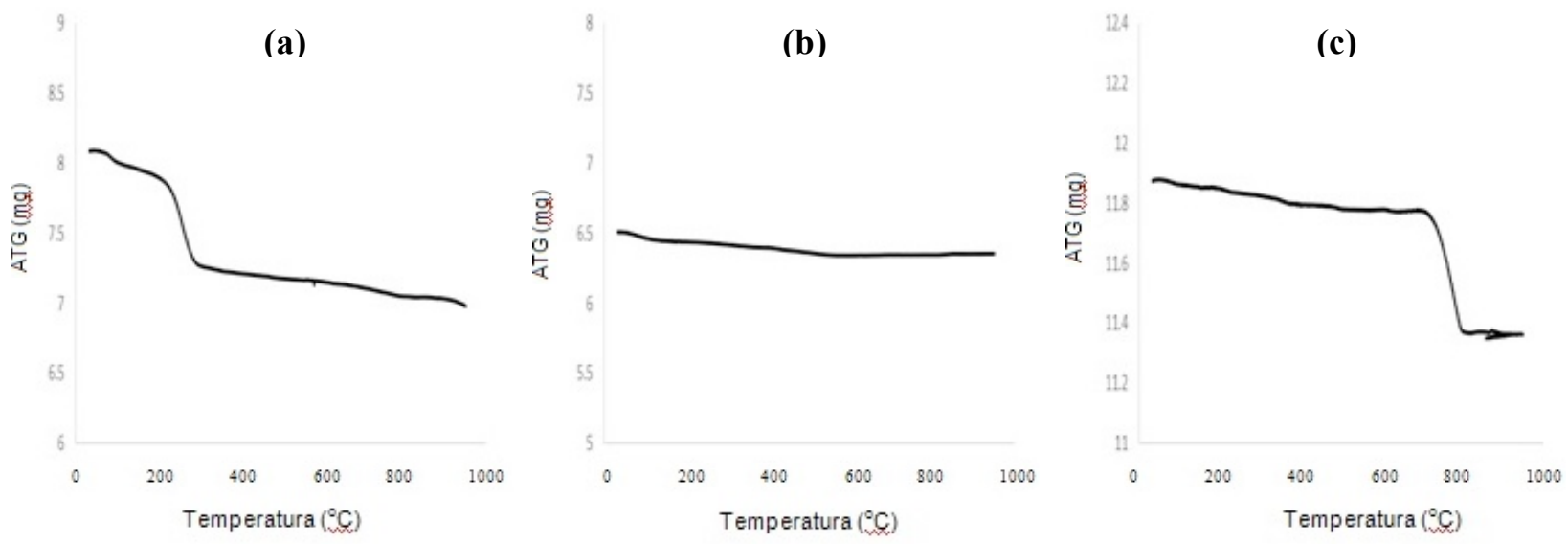

Figura 3 - Análise de ATG dos catalisadores: (a) Goetita, (b) $\mathrm{CeO}_{2}$ e (c) $\mathrm{Mn}_{2} \mathrm{O}_{3}$

\subsection{Tratamento de efluente sintético de refinaria por ozonização catalítica e não catalítica}

Para investigar a eficiência da remoção de COT e DQO, foram realizados dois experimentos: ozonização não catalítica e ozonização catalítica.

Como mostrado na Figura 4a, a concentração de COT diminui à medida que aumenta o tempo de reação em ambos os processos. Porém, a reação de ozonização não catalítica apresentou uma remoção de COT de cerca de $50 \%$, enquanto que, na ozonização catalítica alcançaram-se remoções de até $80 \%$ no mesmo tempo de reação. Evidenciando assim, a eficiência de remoção de matéria orgânica utilizando catalisadores de metais não nobres. Na Figura $4 b$, pode-se observar a remoção de DQO entre os diferentes catalisadores.

As remoções de COT e DQO encontradas para os catalisadores mostram que a goetita foi ligeiramente mais ativa na remoção de COT e DQO, alcançando mais de $78 \%$ de remoção de COT e cerca de $93 \%$ de DQO em 5 horas de tratamento, e este comportamento poderia estar relacionado ao fato da goetita apresentar uma maior área superficial específica BET, a qual hidratada apresenta sítios ativos que auxiliam a geração de mais radicais hidroxilas, aumentando assim, a taxa de mineralização (Kasprzyk-Hordern et al., 2003). Outro aspecto a se considerar é a possibilidade da formação de oxigênio singlete $\left(\mathrm{O}^{*}\right)$, descrita na literatura por Han et al. (2011), que também desempenham um papel importante em processos de oxidação avançada. Como mostrado na análise XPS, a quantidade $\mathrm{O}(1 \mathrm{~s})$ na superfície dos óxidos utilizados neste trabalho decresce na seguinte ordem: goetita $>\mathrm{CeO}_{2}>\mathrm{Mn}_{2} \mathrm{O}_{3}$, que é aproximadamente a mesma ordem de atividade catalítica. $\mathrm{O}$ catalisador $\mathrm{Mn}_{2} \mathrm{O}_{3}$ alcançou $77 \%$ de remoção de COT e $88 \%$ de remoção da DQO, enquanto que o $\mathrm{CeO}_{2}$ resultou em cerca de $80 \%$ de remoção de COT, após 5 horas de reação. 

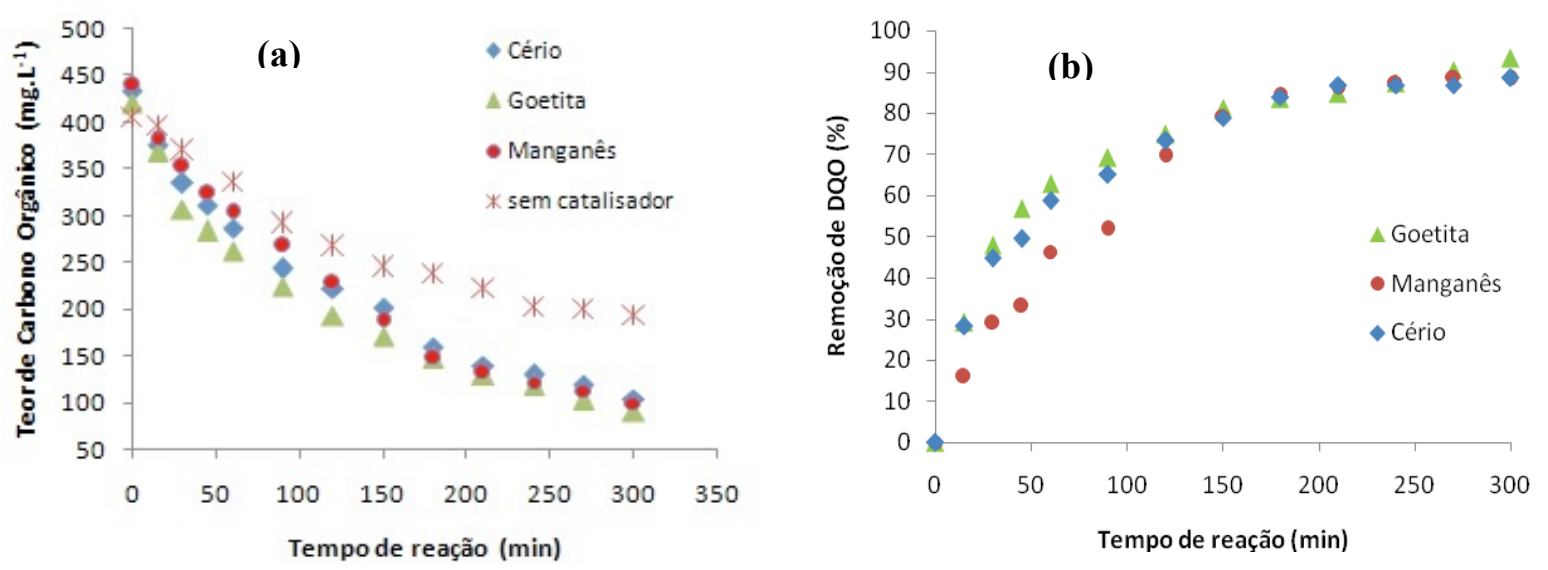

Figura 4 - Cinética de degradação catalítica e não catalítica do ERS (Vazão de ozônio $=0,064 \mathrm{~m}^{3}$ $\mathrm{h}^{-1} ; \mathrm{T}=25^{\circ} \mathrm{C}$; [catalisador] $\left.=0,5 \mathrm{~g} \cdot \mathrm{L}^{-1} ; \mathrm{pH}=7,0\right)$. (a) Teor de TOC $\left(\mathrm{mg} \mathrm{L}^{-1}\right)$ e (b) Redução da DQO (\%).

\section{CONCLUSÃO}

A caracterização das nanopartículas é uma etapa importante, pois permite a avaliação do desempenho dos mesmos como catalisadores. Assim, fatores como, área superficial e morfologia, dentre outros, influenciaram diretamente a eficiência catalítica destes materiais, no processo de ozonização catalítica heterogênea, onde as propriedades oxidativas do ozônio são combinadas com as propriedades adsortivas dos catalisadores, levando a um aumento na taxa de mineralização dos poluentes orgânicos.

Os catalisadores, goetita, $\mathrm{Mn} 2 \mathrm{O} 3 \mathrm{e} \mathrm{CeO}_{2}$, mostraram-se eficientes durante as reações de ozonização, apresentando boa cinética de mineralização, alcançando altas remoções de COT e DQO em $5 \mathrm{~h}$ de tratamento, utilizando uma baixa dosagem de catalisador em suspensão $\left(0,5 \mathrm{~g} \mathrm{~L}^{-}\right.$ $\left.{ }^{1}\right)$. Através da comparação das reações de ozonização catalítica e não catalítica, pôde-se observar que, a degradação de COT aumentou em até $80 \%$ com o uso dos nanocatalisadores.

Assim, o uso de catalisadores na reação de ozonização torna-se importante no aumento da degradação de compostos recalcitrantes, e os catalisadores de óxidos de ferro, manganês ou cério apresentaram aproximadamente a mesma atividade catalítica.

\section{REFERÊNCIAS}

ALMEIDA, E.; ASSALIN, R.; ROSA, M. A. Tratamento de efluentes industriais por processos oxidativos na presença de ozônio. Quím. Nova, v.27, p. 818-824, 2004.

BARBOSA, F. L. Regulamentação do reuso de água em refinarias - análise do modelo americano e perspectivas para o cenário nacional. Dissertação de Mestrado, COPPE/UFRJ, Rio de Janeiro, RJ, 2007. 


\section{9 a 22 de outubro de 2014 \\ Florianópolis/SC}

BIESINGER, M.C.; PAYNE, B.P.; GROSVENOR, A.P.; LAU, L.W.M.; GERSON, A.R.; SMART, R.S.C. Resolving surface chemical states in XPS analysis of first row transition metals, oxides and hydroxides: Cr, Mn, Fe, Co and Ni. Applied Surf.Sci., v. 257, p. 2717-2730, 2011.

BRITTO, J. M.; RANGEL, M. C. Processos avançados de oxidação de compostos fenólicos em efluentes industriais. Quím. Nova, v. 31, p. 114-122, 2008.

COELHO, A.; CASTRO, A.V.; DEZOTTI, M.; SANT'ANNA, G.L. Treatment of petroleum refinery sour water by advanced oxidation processes. J. Hazard.Mater., v. 137, p. 178-184, 2006.

CORNELL, R. M.; SCHWERTMANN, U. The iron oxides. Structure, properties, reaction, occurences and uses. Wiley-VCI, 2003.

DING, Y.; SHEN, X.; SITHAMBARAM, S.; GOMEZ, S.; KUMAR, R.; CRISOSTOMO, V. M. B.; SUIB, S.; AINDOW, M. Synthesis and catalalytic activity of cryptomelane-type manganese dioxide nanomaterials produced by a novel solvent-free method. Chem. Mater., v. 17, p. 53825389, 2005.

DIYA'UDDEEN, B. H.; DAUD, W. M. A. W.; AZIZ, A. R. A. Treatment technologies for petroleum refinery effluents: A review. Process Saf. Environ. Prot., v.89, p. 95-105, 2011.

HAN, S. K.; HWANG, T.-M.; YOON, Y.; KANG, J.-W., Evidence of singlet oxgygen and hydroxyl radical formation in aqueous goethite suspension using spin-trapping electron paramagnetic resonance (EPR), Chemosphere, v. 84, p. 1095-1101, 2011.

IUPAC, Recommendations for the characterization of porous solids. Pure Appl.Chem., v. 66, p. 1739-1758, 1994.

KASPRZYK-HORDERN, B.; ZIÓLEK, M.; NAWROCKI, J. Catalytic ozonation and methods of enhancing molecular ozone reactions in water treatment. Appl. Catal. B: Environ., v. 46, p. 639-669, 2003.

LIN, C. K.; TSAI, T. Y.; LIU, J. C.; CHEN, M. C. Enhanced biodegradation of petrochemical wastewater using ozonation and bac advanced treatment system. Water Res., v. 35, n. 3, p. 699704, 2001.

MARIANO, J. B. Impactos Ambientais do Refino de Petróleo. Dissertação de Mestrado, COPPE/UFRJ, Rio de Janeiro, 2001.

MULLINS, D. R.; OVERBURY, S. H.; HUNTLEY, D. R. Electron spectroscopy of single crystal and polycrystalline cerium oxide surfaces. Surface Sci., v. 409, p. 307-319, 1998.

MUSTAFA, G. Reutilização de Efluentes Líquidos em Indústria Petroquímica. Dissertação de Mestrado, UFBA, Salvador, Bahia, 1998.

NOGUEIRA, M. R. C. Tratamento de efluentes líquidos através de adsorção e oxidação catalítica utilizando catalisadores mistos de ferro e manganês. Dissertação de Mestrado, PPGEQ-UFSC, p. 85, 2009.

PARK, J. S.; CHOI, H.; CHO, J. Kinetic decomposition of ozone and para-chlorobenzoicacid (pCBA) during catalytic ozonation. Water Res., v.38, p. 2285-22. 